\title{
Diagnostic usefulness of KL-6 concentration of bile in biliary tract cancer
}

\author{
TAKUMI ONOYAMA, KAZUYA MATSUMOTO, HIROKI KODA, TARO YAMASHITA, HIROKI KURUMI, \\ SOICHIRO KAWATA, YOHEI TAKEDA, KENICHI HARADA, KAZUO YASHIMA and HAJIME ISOMOTO \\ Division of Medicine and Clinical Science, Department of Multidisciplinary Internal Medicine, \\ Faculty of Medicine, Tottori University, Yonago 683-8504, Japan
}

Received September 9, 2017; Accepted November 28, 2017

DOI: $10.3892 / \mathrm{mco} .2018 .1571$

\begin{abstract}
The sensitivity of bile cytology for biliary tract cancer varies from 6-64\%, and hence remains unsatisfactory. Sialylated carbohydrate antigen KL-6 mucin is positive in biliary tract cancer tissues and serum KL-6 levels are significantly increased in intrahepatic ductal adenocarcinoma patients compared with healthy individuals. The aim of the present study was to evaluate the usefulness of the KL- 6 concentration of bile for the diagnosis of biliary tract cancer. Bile cytology and measurements of bile KL-6 concentration were conducted for 43 patients (25 biliary tract cancers and 18 benign biliary disease). The concentration of KL-6 in the bile of the biliary tract cancer group was compared with the benign biliary disease group. The diagnostic ability was assessed by using receiver operating characteristic curves (ROC). The mean KL- 6 concentration of bile for biliary tract cancer $(34.6 \pm 51.6 \mathrm{U} / \mathrm{ml})$ was increased compared with benign biliary disease $(5.2 \pm 3.9 \mathrm{U} / \mathrm{ml}, \mathrm{P}<0.001)$. The area under the ROC for diagnosis of biliary tract cancer was 0.84 for benign biliary disease. When the cut-off level of the KL-6 concentration of bile was $8.6 \mathrm{U} / \mathrm{ml}$, the sensitivity, specificity, and accuracy of the KL- 6 concentration of bile alone for the diagnosis of biliary tract cancer were 72,89 , and $79 \%$, respectively. Adding the bile KL- 6 concentration to bile cytology measurements, the sensitivity for the diagnosis of biliary tract cancer was increased significantly $(100 \%, \mathrm{P}=0.0184)$. The KL-6 concentration of bile may strengthen the sensitivity of bile cytology for biliary tract cancer.
\end{abstract}

\section{Introduction}

Biliary tract cancer has a poor prognosis because it is often diagnosed at an advanced stage and is often unresectable.

Correspondence to: Dr Kazuya Matsumoto, Division of Medicine and Clinical Science, Department of Multidisciplinary Internal Medicine, Faculty of Medicine, Tottori University, Nishi-machi 36-1, Yonago 683-8504, Japan

E-mail: matsumot@med.tottori-u.ac.jp

Key words: KL-6, biliary tract cancer, bile cytology
If an early diagnosis of this malignancy is possible, the prognosis might improve (1). Again, it is often difficult to differentiate malignant biliary tract strictures and benign biliary strictures: Such as primary sclerosing cholangitis, IgG4-associated sclerosing cholangitis and Mirrizi syndrome (2). It is important to distinguish biliary tract cancer from benign biliary disease because the treatment strategies and prognoses differ. In patients with a biliary stricture, endoscopic retrograde cholangiopancreatography (ERCP) is a common pathological diagnostic method that allows the use of several techniques for tissue sampling, including bile aspiration cytology, brush cytology, and forceps biopsy. The specificities of the pathological examination of tissue obtained by ERCP for biliary strictures are almost $100 \%$, thus, obtaining histological or cytological evidence is very important to determine the therapeutic strategies in these patients. However the sensitivities of bile aspiration cytology, brush cytology, and forceps biopsy for biliary strictures are $6-72 \%(3,4)$, have not been satisfactory. Therefore, improvement of the sensitivity for diagnosing biliary tract cancer is needed.

Mucins, which are produced by various epithelial cells, are high molecular weight glycoproteins with oligosaccharides attached to serine or threonine residues of the mucin core protein backbone by O-glycosidic linkages. The human mucin (MUC) family consists of members designated MUC1 to MUC21. Mucins are also expressed in pancreatico-biliary neoplasm included biliary tract cancer. Furthermore, MUC expression pattern might reflect the cell differentiation type of biliary tract cancer like pancreatic intraductal papillary mucinus neoplasms, for example, gastric type presents a MUC1-/MUC2-/MUC5AC+/MUC6-profile,intestinaltypepresentsa MUC1-/MUC2+/MUC5AC+/MUC6-profile, and pancreaticobiliary type presents a MUC1+/MUC2-/MUC5AC+/MUC6+ profile $(5,6)$.

Sialylated carbohydrate antigen KL-6, a type of Mucin 1, cell surface associated (MUC1), was investigated and was suggested to have a significant relationship with a worse tumor behavior, especially cancer cell invasion and metastasis in gastrointestinal, hepatic, pancreatico-biliary and ampullary cancers (7-9). Although the KL-6 concentration of serum for intrahepatic ductal adenocarcinoma and pancreatic juice for pancreatic ductal adenocarcinoma was useful 
in diagnosing pancreatic ductal adenocarcinoma (10-12), there have not been any studies about the clinical benefits of measuring the KL-6 concentration of bile for diagnosing biliary tract cancer. In the present study, the usefulness of the KL- 6 concentration of bile for diagnosis of biliary tract cancer was examined.

\section{Patients and methods}

A total of 43 patients with biliary disease were enrolled prospectively between October 2011 and January 2014 at our hospital. The diagnosis of biliary tract cancer was based on the pathological diagnosis of bile aspiration cytology, transpapillary forceps biopsy, endoscopic ultrasound fine needle aspiration (EUS-FNA) or surgical specimen. Patients without a malignant disease had a final benign diagnosis based on clinical and radiological follow-up data for at least 6 months. This study was performed according to the guidelines described in the Helsinki Declaration for biomedical research involving human subjects. The study protocol was approved by the institutional review board of Tottori University. Written informed consent was obtained from all participating subjects.

The 43 patients with biliary disease included 28 men and 15 women: Age range, 34-88 years; mean age, 72.6 years (Table I). A malignant lesion was present in 25 patients and a benign lesion was present in 18 patients.

We performed ERCP and transpapillary bile aspiration cytology. Cytodiagnosis of the specimens was performed by Papanicolaou's method. A lateral-viewing duodenoscope (JF260V; Olympus Optical Co., Ltd, Tokyo, Japan) was used to carry out ERCP. Bile was collected by aspirating through a biliary catheter from the bile duct during ERCP by using a cannula (M00535700; Boston Scientific Corporation, Natick, MA, USA), and a 0.035-inch hydrophilic guide-wire (M00556051; Boston Scientific Corporation). Over the guide-wire, the cannula was advanced into the bile duct. The guide-wire was then withdrawn, and bile was collected using a syringe with the tip of the cannula in the bile duct. The aspirated specimen was then evaluated.

Afterwards, the bile was centrifuged at $1,710 \mathrm{xg}$ for $5 \mathrm{~min}$, the pellet was subjected to cytological examination, and a supernatant was used for measuring the KL-6 concentration. Human KL-6 levels were determined in duplicate with a PICOLUMI KL-6 kit (EIDIA, Tokyo, Japan), and an electrochemiluminescence immunoassay (ECLIA) specific for human KL-6.

We compared the KL-6 concentration of bile in the biliary tract cancer group with the benign biliary disease group. We also evaluated the utility of measuring the KL- 6 concentration of bile for use in the diagnosis of biliary tract cancer.

Statistical analysis. The statistical analysis was performed using StatFlex ver. 6.0 for Windows software (Artech Co, Ltd., Osaka, Japan). Categorical variables were compared by using the Chi-square test. Continuous variables were compared by using the Mann-Whitney $U$-test. Comparisons of the mean KL-6 concentration of bile between biliary tract cancer and benign biliary disease were performed by using the Mann-Whitney $U$-test. All values are expressed as means \pm standard deviation or means with interquartile ranges. $\mathrm{P}<0.05$ was considered

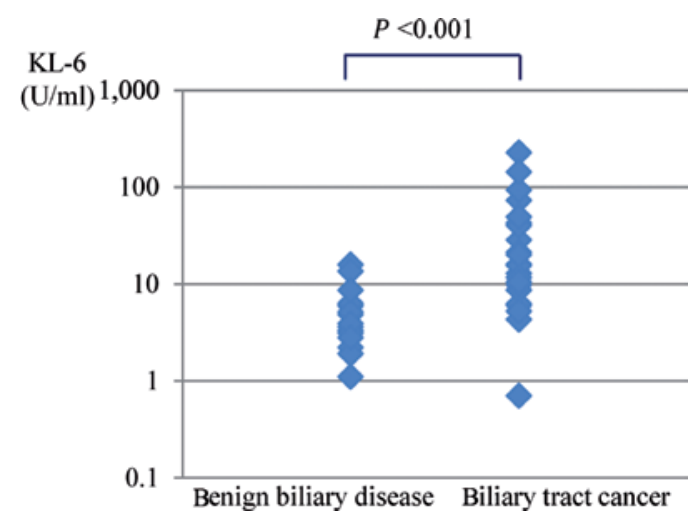

Figure 1. The KL-6 concentration of bile in biliary diseases. The mean of bile KL-6 concentration was significantly higher for biliary tract cancer $(34.6 \pm 51.6 \mathrm{U} / \mathrm{ml})$ than for benign biliary disease $(5.2 \pm 3.9 \mathrm{U} / \mathrm{ml}, \mathrm{P}<0.001)$.

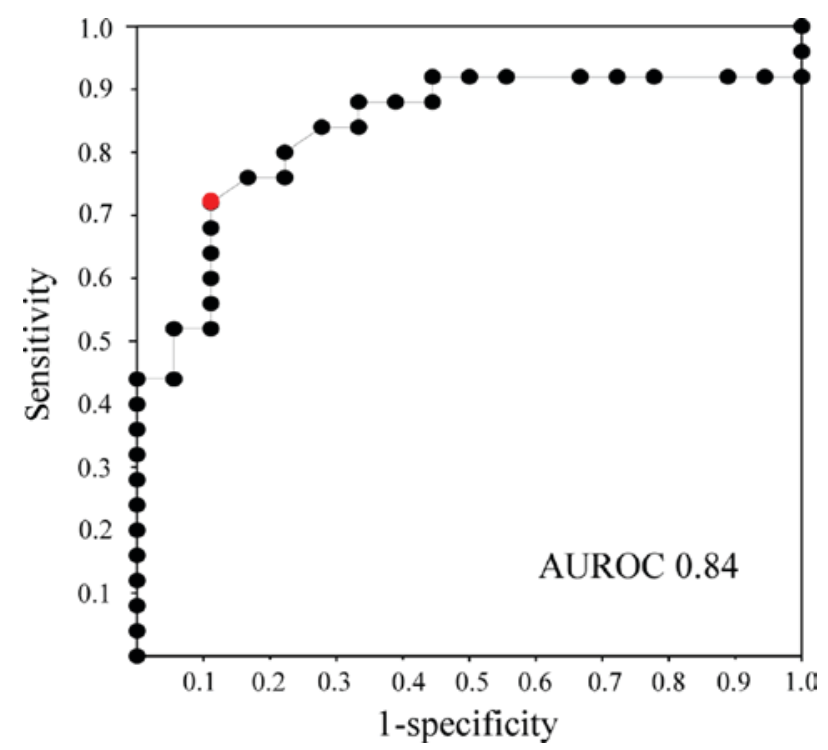

Figure 2. Receiver operating characteristic (ROC) curve of the KL-6 concentration of bile for biliary tract cancer. According to the ROC curve, the optical cut-off value of bile KL- 6 for distinguishing biliary tract cancer from benign biliary disease is estimated to be $8.6 \mathrm{U} / \mathrm{ml}$. The area under the receiver operating characteristic curve (AUROC) of the bile KL-6 analysis is 0.84 .

significant. The diagnostic power of KL-6 concentration of bile was assessed by using a receiver-operating characteristic (ROC) curves analysis. Optimal cut-off levels for KL-6 of bile were determined according to Youden index, and positive and negative predictive values were evaluated using these cut-off values.

\section{Results}

The patients with biliary disease characteristics are shown in Table I. The malignant group included 11 perihilar bile duct adenocarcinoma, 8 distal bile duct adenocarcinoma and 6 gallbladder adenocarcinoma. The growth pattern types of biliary tract cancer were 4 mass-forming type, 9 intraductal type and 12 periductal type, respectively. The benign group included 9 benign biliary strictures, 1 pancreaticobiliary maljunction, 2 chronic cholecystitis, 1 adenomyomatosis of gallbladder, 3 adenoma of the papilla and 2 papillitis of Vater.

There was no significant difference in age and sex between the malignant group and the benign group. The median level 
Table I. Patients' characteristics with benign biliary disease and patients with biliary tract cancer.

\begin{tabular}{|c|c|c|c|}
\hline & Benign biliary disease $(n=18)$ & Biliary tract cancer $(n=25)$ & P-value \\
\hline Mean age, year & $69.8(42-88)$ & $74.6(51-87)$ & $0.07^{\mathrm{a}}$ \\
\hline Number of patients $(\mathrm{M} / \mathrm{F})$ & $18(13 / 5)$ & $25(15 / 10)$ & $0.41^{\mathrm{b}}$ \\
\hline Mean size of tumor, mm & - & $36.3(7-100)$ & - \\
\hline \multicolumn{4}{|l|}{ Tumor marker (serum) } \\
\hline CEA, ng/ml & $4.2(1.2-13.5)$ & $20.6(0.8-337.7)$ & $0.979^{a}$ \\
\hline CA19-9, U/ml & $36.6(3.7-187.8)$ & $483.0(4.7-4939)$ & $<0.01^{\mathrm{a}}$ \\
\hline $\mathrm{KL}-6, \mathrm{U} / \mathrm{ml}$ & $260.5(133-412)$ & $830.7(144-8176)$ & $0.16^{\mathrm{a}}$ \\
\hline
\end{tabular}

of serum CA19-9 in the malignant group was significantly higher than that in the benign group. There were no significant differences in the level of serum CEA and KL- 6 between the malignant group and the benign group.

The KL-6 concentration of bile in the malignant group and the benign group are shown in Fig. 1. The average KL-6 concentration of bile was significantly higher for biliary tract cancer $(34.6 \pm 51.6 \mathrm{U} / \mathrm{ml})$ than for benign biliary disease (5.2 $\pm 3.9 \mathrm{U} / \mathrm{ml}, \mathrm{P}<0.001)$.

The ROC curves for bile KL- 6 concentration showed that the area under the receiver operating characteristic curve (AUROC) had a value of 0.84 between biliary tract cancer and benign biliary disease (Fig. 2). According to the ROC curves, the cut-off value of bile KL- 6 for the diagnosis of biliary tract cancer was estimated to be $8.6 \mathrm{U} / \mathrm{ml}$.

Compared with bile KL- 6 in patients with benign biliary disease, the cut-off value of bile KL-6 for the diagnosis of biliary tract cancer was associated with a sensitivity, specificity, positive predictive values, negative predictive values, and accuracy of $72,89,90,70$ and $79 \%$, respectively (Tables II and III).

Table IV summarizes the diagnostic ability of bile aspiration cytology and/or KL-6 analysis to differentiate biliary tract cancer from benign biliary disease. The sensitivity, specificity, positive predictive value, negative predictive value, and accuracy of bile aspiration cytology alone were 80, 100, 100, 78 , and $88 \%$, respectively. Of the remaining 5 patients who remained undiagnosed by cytological assessment, the KL-6 concentration of bile was measured in all $5(100 \%)$ patients. Adding the KL-6 concentration of the bile aspiration cytology diagnosis significantly increased the sensitivity of bile aspiration cytology by $20.0 \%(\mathrm{P}=0.018)$.

Six patients $(14.0 \%)$ in this study developed complications following bile aspiration cytology during ERCP. Mild pancreatitis occurred at a rate of $9.3 \%$ (4/43) and cholangitis occurred at a rate of $4.7 \%(2 / 43)$. All were resolved with conservative treatment. No serious complications such as perforation or hemorrhages were observed. There was no procedure related mortality.

\section{Discussion}

Although ERCP plays an important role in the diagnosis for biliary stricture, the sensitivity is not enough. Recent studies showed that the sensitivity of bile aspiration cytology, biliary brush cytology and forceps biopsy for malignant biliary strictures were $41.6,45.0$, and $48.1 \%$, respectively. A combination of both modalities only modestly increased the sensitivity to $59.4 \%$. Both techniques are almost $100 \%$ specific $(3,4)$. Furthermore, the incidence rates of post-ERCP complication, which were reported as 4.0-6.9\%, including pancreatitis (2.6-3.5\%), bleeding (0.3-1.3\%), and perforation (0.1-0.6\%), can not be ignored $(13,14)$.

Recently, the high sensitivity (80\%) and the low complication rate (bleeding $1.0 \%$, biliary peritonititis $0.3 \%$ ) of EUS-FNA for the diagnosis of malignant biliary strictures were reported (15-17). Meanwhile small lesions, especially the lesions present with wall thickening, are more difficult to sample by using EUS-FNA. In addition, the possibility for needle tract seeding in resectable cases is unresolved (18).

ERCP is a common method for tissue sampling in patients with biliary strictures by bile aspiration cytology, biliary brush cytology and forceps biopsy. A recent study showed that the sensitivity and accuracy of bile aspiration cytology for malignant biliary strictures were 41.6 and $67.7 \%$, respectively (3). Navaneenthan et al reported that the sensitivities of transpapilary brush cytology and forceps biopsy in diagnosing malignant biliary strictures were 45.0 and $48.1 \%$, respectively. A combination of both modalities only modestly increased the sensitivity to $59.4 \%$. Both techniques are almost $100 \%$ specific (4). Bile aspiration cytology is easier and safer than brush cytology and forceps biopsy, which are technically difficult and carry some degree of complication. Therefore, bile aspiration cytology during ERCP is an effective method for the cytological diagnosis of biliary tract cancer, although the sensitivity is inadequate.

Tang et al reported that KL-6 mucin, one kind of MUC1, was positive in biliary tract cancer tissues (10). Xu et al also reported KL-6 might be involved in tumor cell adhesion and invasion in intrahepatic cholangiocarcinoma (9). These reports mean that there is the potential for an increase in bile KL-6 concentration in patients with biliary tract cancer. So, we considered the KL-6 concentration of bile measurement in patients with biliary disease might be helpful to diagnose biliary tract cancer.

In the current study, a high KL-6 concentration of bile was seen in $72 \%$ of patients with biliary tract cancer. The sensitivity of bile aspiration cytology for the diagnosis of biliary tract 
Table II. The cut-off levels of bile KL-6 for biliary tract cancer.

\begin{tabular}{|c|c|c|c|c|c|c|c|}
\hline Cut-off & Sensitivity & 1-Specificity & PPV & NPV & Lielihood ratio & Odds ratio & Youden-index \\
\hline 225.100 & 0.0000 & 0.0000 & - & 0.4186 & - & - & 0.0000 \\
\hline 142.400 & 0.0400 & 0.0000 & 1.0000 & 0.4286 & - & - & 0.0400 \\
\hline 92.600 & 0.0800 & 0.0000 & 1.0000 & 0.4390 & - & - & 0.0800 \\
\hline 72.500 & 0.1200 & 0.0000 & 1.0000 & 0.4500 & - & - & 0.1200 \\
\hline 49.000 & 0.1600 & 0.0000 & 1.0000 & 0.4615 & - & - & 0.1600 \\
\hline 42.200 & 0.2000 & 0.0000 & 1.0000 & 0.4737 & - & - & 0.2000 \\
\hline 40.000 & 0.2400 & 0.0000 & 1.0000 & 0.4865 & - & - & 0.2400 \\
\hline 28.300 & 0.2800 & 0.0000 & 1.0000 & 0.5000 & - & - & 0.2800 \\
\hline 21.200 & 0.3200 & 0.0000 & 1.0000 & 0.5143 & - & - & 0.3200 \\
\hline 20.000 & 0.3600 & 0.0000 & 1.0000 & 0.5294 & - & - & 0.3600 \\
\hline 15.900 & 0.4000 & 0.0000 & 1.0000 & 0.5455 & - & - & 0.4000 \\
\hline 15.800 & 0.4400 & 0.0000 & 1.0000 & 0.5625 & - & - & 0.4400 \\
\hline 15.400 & 0.4400 & 0.0000 & 1.0000 & 0.5484 & 7.9200 & 13.3571 & 0.4400 \\
\hline 13.500 & 0.5200 & 0.0556 & 0.9286 & 0.5862 & 9.3600 & 18.4167 & 0.4644 \\
\hline 12.800 & 0.5200 & 0.1111 & 0.8667 & 0.5714 & 4.6800 & 8.6667 & 0.4089 \\
\hline 11.600 & 0.5600 & 0.1111 & 0.8750 & 0.5926 & 5.0400 & 10.1818 & 0.4489 \\
\hline 10.400 & 0.6000 & 0.1111 & 0.8824 & 0.6154 & 5.4000 & 12.0000 & 0.4889 \\
\hline 9.800 & 0.6400 & 0.1111 & 0.8889 & 0.6400 & 5.7600 & 14.2222 & 0.5289 \\
\hline 8.700 & 0.6800 & 0.1111 & 0.8947 & 0.6667 & 6.1200 & 17.0000 & 0.5689 \\
\hline 8.600 & 0.7200 & 0.1111 & 0.9000 & 0.6957 & 6.4800 & 20.5714 & 0.6089 \\
\hline 6.300 & 0.7600 & 0.1667 & 0.8636 & 0.7143 & 4.5600 & 15.8333 & 0.5933 \\
\hline 6.200 & 0.7600 & 0.2222 & 0.8261 & 0.7000 & 3.4200 & 11.0833 & 0.5378 \\
\hline 6.000 & 0.8000 & 0.2222 & 0.8333 & 0.7368 & 3.6000 & 14.0000 & 0.5778 \\
\hline 5.900 & 0.8400 & 0.2778 & 0.8077 & 0.7647 & 3.0240 & 13.6500 & 0.5622 \\
\hline 5.200 & 0.8400 & 0.3333 & 0.7778 & 0.7500 & 2.5200 & 10.5000 & 0.5067 \\
\hline 5.100 & 0.8800 & 0.3333 & 0.7857 & 0.8000 & 2.6400 & 14.6667 & 0.5467 \\
\hline 4.800 & 0.8800 & 0.3889 & 0.7586 & 0.7857 & 2.2629 & 11.5238 & 0.4911 \\
\hline 4.300 & 0.8800 & 0.4444 & 0.7333 & 0.7692 & 1.9800 & 9.1667 & 0.4356 \\
\hline 3.900 & 0.9200 & 0.4444 & 0.7419 & 0.8333 & 2.0700 & 14.3750 & 0.4756 \\
\hline 3.600 & 0.9200 & 0.5000 & 0.7186 & 0.8182 & 1.8400 & 11.5000 & 0.4200 \\
\hline 3.300 & 0.9200 & 0.5556 & 0.6970 & 0.8000 & 1.6560 & 9.2000 & 0.3644 \\
\hline 3.100 & 0.9200 & 0.6667 & 0.6571 & 0.7500 & 1.3800 & 5.7500 & 0.2533 \\
\hline 2.800 & 0.9200 & 0.7222 & 0.6389 & 0.7143 & 1.2739 & 4.4231 & 0.1978 \\
\hline 2.200 & 0.9200 & 0.7778 & 0.6216 & 0.6667 & 1.1829 & 3.2857 & 0.1422 \\
\hline 1.900 & 0.9200 & 0.8889 & 0.5897 & 0.5000 & 1.0350 & 1.4375 & 0.0311 \\
\hline 1.100 & 0.9200 & 0.9444 & 0.5750 & 0.3333 & 0.9741 & 0.6765 & -0.0244 \\
\hline 0.700 & 0.9200 & 1.0000 & 0.5610 & 0.0000 & 0.9200 & 0.0000 & -0.0800 \\
\hline 0.000 & 0.9600 & 1.0000 & 0.5714 & 0.0000 & 0.9600 & 0.0000 & -0.0400 \\
\hline
\end{tabular}

PPV, positive predictive value; NPV, negative predictive value.

Table III. Diagnostic ability of KL-6 measurement of bile for differentiating biliary tract cancer from benign biliary disease.

\begin{tabular}{|c|c|c|c|c|c|c|}
\hline & Optimal cut-off values, $\%$ & Sensitivity, $\%$ & Specificity, \% & PPV, \% & NPV, $\%$ & Accuracy, \% \\
\hline KL-6, U/ml & 8.6 & 72 & 89 & 90 & 70 & 79 \\
\hline
\end{tabular}

cancer was significantly improved by adding the bile KL-6 concentration although bile aspiration cytology showed favorable sensitivity for biliary tract cancer in the present study. If the sensitivity of bile aspiration cytology for biliary 
Table IV. Diagnostic ability of bile aspiration cytology and/or KL-6 measurement of bile for differentiating biliary tract cancer from benign biliary disease.

\begin{tabular}{|c|c|c|c|c|c|}
\hline & Sensitivity, $\%$ & Specificity, \% & PPV, \% & NPV, \% & Accuracy, $\%$ \\
\hline \multirow[t]{2}{*}{ KL-6 measurement } & 72 & 89 & 90 & 70 & 79 \\
\hline & $(18 / 25)$ & $(16 / 18)$ & $(18 / 20)$ & $(16 / 23)$ & $(34 / 43)$ \\
\hline \multirow[t]{2}{*}{ Bile aspiration cytology } & 80 & 100 & 100 & 78 & 88 \\
\hline & $(20 / 25)$ & $(18 / 18)$ & $(20 / 20)$ & $(18 / 23)$ & $(38 / 43)$ \\
\hline Bile aspiration cytology & $100^{\mathrm{a}}$ & 89 & 93 & 100 & 95 \\
\hline And/or KL-6 measurement & $(25 / 25)$ & $(16 / 18)$ & $(25 / 27)$ & $(15 / 15)$ & $(41 / 43)$ \\
\hline
\end{tabular}

PPV, positive predictive value; NPV, negative predictive value. P-value: $\chi^{2}$ test ${ }^{\mathrm{P}}=0.018$ compared with cytology alone.

tract cancer was as low as that of ERCP-guided tissue sampling in recent studies, the measurement of bile KL-6 concentration might have improved the diagnostic ability of bile cytology for biliary tract cancer more. Biliary tract cancer whose bile cytology results were inconclusive or negative could be diagnosed exactly by combining the bile KL-6 measurements with the bile cytology results. Because the high-accuracy of bile aspiration cytology for biliary tract cancer was evaluated in the present study, the diagnostic ability of the combination of bile aspiration cytology and bile KL-6 concentration was not significantly higher than that of bile aspiration cytology only. We thought that the sensitivity of bile KL-6 concentration might be better than that of bile aspiration cytology if the diagnostic ability of bile aspiration cytology for biliary tract cancer was low. However, a high concentration of bile KL-6 was also seen in $11 \%$ of patients with benign biliary disease because the specificity of KL-6 of bile was inadequate. These findings suggest that further examinations such as trasnpapilary brush cytology, forceps biopsy, cholangioscopy, and EUS-FNA are necessary when bile aspiration cytology specimens are negative and the KL- 6 concentration of bile is increased.

The other benefit of the measurement of bile KL-6 is that it does not affect the diagnostic ability of bile aspiration cytology because the KL- 6 concentration was evaluated by using the supernatant of bile from which the cell pellet was removed for cytological examination.

There were no significant differences in the level of serum KL-6 between the biliary tract cancer group and the benign biliary disease group in this study although the elevation of serum KL-6 mucin levels in patients with cholangiocarcinoma was reported in previous study (11). The reason of this discrepancy might be involved in MUC1 gene polymorphisms which are associated with serum KL-6 levels (19). The association between bile KL-6 and MUC1 gene polymorphisms was uncertain, more study is needed.

The present study has some limitations. Firstly, this study was a single-center study with small number of cases. Secondly, the in-vivo and in-situ experiments of KL-6 were not evaluated. Thirdly, this sample size is insufficient to conclude that KL-6 as a diagnostic factor in biliary tract cancers. Fourthly, if we checked KL-6 level by using samples from cancer adjunct tissue, we might improve the accuracy of the central conclusion. Finally, other biliary tract neoplasms, such as neuroendocrine tumors, and para-biliary malignant tumors which may cause biliary strictures, such as pancreatic ductal adenocarcinoma were not evaluated.

In conclusion, the KL- 6 concentration of bile may strengthen the sensitivity of bile cytology for biliary tract cancer.

\section{References}

1. Ishihara S, Horiguchi A, Miyakawa S, Endo I, Miyazaki M and Takada T: Biliary tract cancer registry in Japan from 2008 to 2013. J Hepatobiliary Pancreat Sci 23: 149-157, 2016.

2. Wakai T, Shirai Y, Sakata J, Maruyama T, Ohashi T, Korira PV, Ajioka Y and Hatakeyama K: Clinicopathological features of benign biliary strictures masquerading as biliary malignancy. Am Surg 78: 1388-1391, 2012.

3. Burnett AS, Calvert TJ and Chokshi RJ: Sensitivity of endoscopic retrograde cholangiopancreatography standard cytology: 10-y review of the literature. J Surg Res 184: 304-311, 2013.

4. Navaneethan U, Njei B, Lourdusamy V, Konjeti R, Vargo JJ and Parsi MA: Comparative effectiveness of biliary brush cytology and intraductal biopsy for detection of malignant biliary strictures: A systematic review and meta-analysis. Gastrointest Endosc 81: 168-176, 2015.

5. Yonezawa S, Higashi M, Yamada N, Yokoyama S and Goto M: Significance of mucin expression in pancreatobiliary neoplasms. J Hepatobiliary Pancreat Sci 17: 108-124, 2010.

6. Moschovis D, Bamias G and Delladetsima I: Mucins in neoplasms of pancreas, ampulla of Vater and biliary system. World J Gastrointest Oncol 8: 725-734, 2016.

7. Inagaki Y, Xu H, Nakata M, Seyama Y, Hasegawa K, Sugawara Y, Tang W and Kokudo N: Clinicopathology of sialomucin: MUC1, particularly KL-6 mucin, in gastrointestinal, hepatic and pancreatic cancers. Biosci Trends 3: 220-232, 2009.

8. Tang W, Inagaki Y, Kokudo N, Guo Q, Seyama Y, Nakata M, Imamura H, Sano K, Sugawara Y and Makuuchi M: KL-6 mucin expression in carcinoma of the ampulla of Vater: Association with cancer progression. World J Gastroenterol 11: 5450-5454, 2005.

9. Xu HL, Inagaki Y, Seyama Y, Sugawara Y, Kokudo N, Nakata M, Wang FS and Tang W: Expression of KL-6 mucin, a human MUC1 mucin, in intrahepatic cholangiocarcinoma and its potential involvement in tumor cell adhesion and invasion. Life Sci 85: 395-400, 2009.

10. Tang W, Guo Q, Qu X, Inagaki Y, Seyama Y, Midorikawa Y, Gai R, Kokudo N, Sugawara Y, Nakata M and Makuuchi M: KL-6 mucin is a useful immunohistochemical marker for cholangiocarcinoma. Oncol Rep 17: 737-741, 2007.

11. Xu H, Inagaki Y, Tang W, Guo Q, Wang F, Seyama Y, Midorikawa Y, Gai R, Kokudo N, Sugawara Y, et al: Elevation of serum KL-6 mucin levels in patients with cholangiocarcinoma. Hepatogastroenterology 55: 2000-2004, 2008.

12. Matsumoto K, Takeda Y, Harada K, Onoyama T, Kawata S, Horie Y, Sakamoto T, Ueki M, Miura N and Murawaki Y: Clinical impact of the KL-6 concentration of pancreatic juice for diagnosing pancreatic masses. Biomed Res Int 2015: 528304, 2015. 
13. Cotton PB, Garrow DA, Gallagher J and Romagnuolo J: Risk factors for complications after ERCP: A multivariate analysis of 11,497 procedures over 12 years. Gastrointest Endosc 70: 80-88, 2009.

14. Andriulli A, Loperfido S, Napolitano G, Niro G, Valvano MR, Spirito F, Pilotto A and Forlano R: Incidence rates of post-ERCP complications: A systematic survey of prospective studies. Am J Gastroenterol 102: 1781-1788, 2007.

15. Sadeghi A, Mohamadnejad M, Islami F, Keshtkar A, Biglari M, Malekzadeh R and Eloubeidi MA: Diagnostic yield of EUS-guided FNA for malignant biliary stricture: A systematic review and meta-analysis. Gastrointest Endosc 83: 290-8.e1, 2016.

16. Weilert F, Bhat YM, Binmoeller KF, Kane S, Jaffee IM, Shaw RE, Cameron R, Hashimoto Y and Shah JN: EUS-FNA is superior to ERCP-based tissue sampling in suspected malignant biliary obstruction: Results of a prospective, single-blind, comparative study. Gastrointest Endosc 80: 97-104, 2014.
17. De Moura DT, Moura EG, Bernardo WM, De Moura ET, Baracat FI, Kondo A, Matuguma SE and Artifon EL: Endoscopic retrograde cholangiopancreatography versus endoscopic ultrasound for tissue diagnosis of malignant biliary stricture: Systematic review and meta-analysis. Endosc Ultrasound: Nov 8 , 2016 (Epub ahead of print).

18. Heimbach JK, Sanchez W, Rosen CB and Gores GJ: Trans-peritoneal fine needle aspiration biopsy of hilar cholangiocarcinoma is associated with disease dissemination. HPB(Oxford) 13: 356-360, 2011.

19. Bonella F, Long X, Ohshimo S, Horimasu Y, Griese M, Guzman J, Kohno N and Costabel U: MUC1 gene polymorphisms are associated with serum KL-6 levels and pulmonary dysfunction in pulmonary alveolar proteinosis. Orphanet J Rare Dis 11: 48, 2016. 